\title{
Genes for Life: Biobanking for Genetic Research in Africa
}

\author{
Elizabeth Sarah Mayne, ${ }^{1}$ Talishiea Croxton, ${ }^{2,3}$ Alash'le Abimiku, 2,3 \\ Moses Joloba, ${ }^{4}$ Samuel Kyobe, ${ }^{4}$ Christine M. Beiswanger, ${ }^{5}$ Louise Wideroff, ${ }^{6}$ Mark Guyer, \\ Jennifer Troyer, ${ }^{6}$ Mukthar Kader $^{7}$; and H3Africa Biorepository Working Group
}

$\mathrm{B}$ IOBANKS ARE ORGANIZED collections of biospecimens and associated data from large number of individuals for the purpose of health research. The number of biobanks has expanded in the genomics era to facilitate analysis of high-quality biospecimens with associated phenotypic data. Biobanks support biomarker identification, therapeutic development, and evidence-based decision making that ultimately benefit public health. ${ }^{1}$

Biobanking in low- and middle-income countries (LMICs) is less developed, with most biobanks in these settings focusing on specific diseases or populations. ${ }^{2}$ Human disease is under-researched in LMICs that carry $90 \%$ of the global disease burden and produce only $10 \%$ of the global research output. $^{3}$ Only $2 \%$ of authors publishing in biobanking are based in Africa. ${ }^{4}$ Many African biobanks only store material temporarily for shipment to higher income countries. 5,6

Human Health and Heredity in Africa (H3Africa) was established as a pan-African Consortium to study the genetic basis of diseases with African (and international) relevance. A mandate of the consortium is capacity development in genetic research, ${ }^{7,8}$ and storage of high-quality DNA and other biological samples is central to accomplishing this mandate. ${ }^{5}$

Although established biobanks exist outside Africa, reasons exist for establishing African biobanks for African research. ${ }^{9,10}$ African populations are genetically diverse. Fully understanding the genetic basis of disease among the different populations, geographically and ethnically, is central to improving healthcare by identifying new targets for diagnosis and therapy. This population-based genetic research requires resources. Of these, high-quality stored DNA with associated phenotypic data is one of the most important. In addition, cultural, ethical, and legal requirements insist that samples collected from African populations contribute to those populations' health needs. ${ }^{11}$ Certain African countries have regulations prohibiting storage of biospecimens for unspecified research, others require separate consent for storage of samples. Approval may also be required for reuse or export of samples. ${ }^{12}$ These requirements mean that clinical researchers must be empowered to design protocols that will accurately reflect both the planned usage of stored material and the local understanding and regulations concerning consent. Custodianship of samples and phenotypic data often rests with the participant rather than the researcher. It is easier to align with national policies when samples remain on the continent. The H3Africa biorepositories are integrally involved in the design of storage guidelines and material transfer agreements in consultation with institutional review boards, national experts, and community leaders. ${ }^{13}$

Biobanks in Africa also face some challenges, including logistical problems, infrastructure requirements, and training needs. A number of African countries have become adept at working within these constraints, but, in alignment with the goals of H3Africa, there has been significant progress in establishing facilities that meet international standards. These adapt technologies for storage of genetic material for the H3Africa project. It often becomes necessary to develop local solutions, for example, hybrid solutions for H3Africa shipment problems. These include consortium-wide negotiations to ensure that couriers provide cost-efficient shipping with effective local service, consideration of alternatives to using dry ice, and communication modalities to maintain and monitor sample chainof-custody. These solutions may inform future development of biorepositories on the continent and in other LMICs. Spare capacity could facilitate collaboration with other stakeholders for current and future genetic research with high-quality genetic specimens for the African scientists trained within the H3Africa consortium. ${ }^{14}$

\footnotetext{
${ }^{1}$ Department of Molecular Medicine and Haemotology, University of the Witwatersrand, National Health Laboratory Service, Johannesburg, South Africa.

${ }^{2}$ Institute of Human Virology-Nigeria, Abuja, Nigeria

${ }^{3}$ University of Maryland, College Park, Maryland.

${ }_{5}^{4}$ Makerere University, Kampala, Uganda.

${ }^{5}$ Coriell Institute for Medical Research, Abuja, Nigeria.

${ }^{6}$ National Institutes of Health, Bethesda, Maryland.

${ }^{7}$ Clinical Laboratory Services, University of the Witwatersrand, Johannesburg, South Africa.
}

(c) Elizabeth Sarah Mayne et al. 2017; Published by Mary Ann Liebert, Inc. This is an Open Access article distributed under the terms of the Creative Commons Attribution License, which permits unrestricted use, distribution, and reproduction in any medium, provided the original work is properly cited. 
Finally, it is important that African health needs are addressed. This is best done with local access to the samples and local research protocols. Local ownership of samples and biobanks is key to ensuring sustainability of the biorepositories. Ownership of the research generated will ensure that findings translate into healthcare practices in the local community, health policies, and regulations. ${ }^{10}$ Storage of biological material from patients with rare diseases, found only in African populations, in addition to large stores of material from patients with common conditions, which pose a significant public health risk, and from healthy controls ${ }^{5,15}$ will enable Africans to participate in global initiatives to counter health threats.

Biobanks are a significant resource for any research initiative. They will be fundamental pillars underpinning the development of world-class research on the African continent. As the importance of storing genetic material is highlighted by the outputs already flowing from the clinical projects in H3Africa, it is hoped that the potential applications of stored material from basic research to quality assurance to informing health policy are recognized by the stakeholders. Biorepositories must store inclusively, representing the widely diverse ethnic populations in Africa, and provide inclusive outputs with long-term benefits for African and international health. Quality specimens stored in the H3Africa biorepositories will be a vital resource for future research to strengthen health systems. The vision of the H3Africa biorepository flagship project is to map the path for African biorepositories so that Africans possess the tools to research and take ownership of their own health.

\section{Acknowledgments}

The H3Africa Consortium Biorepositories are funded by the U.S. National Institutes of Health grants UH3HG007008, UH3HG007051, UH3HG007438, UH2HG007092 of the H3Africa Common Fund Initiative.

\section{Author Disclosure Statement}

No conflicting financial interests exist.

\section{References}

1. Fransson $\mathrm{MN}$, et al. Toward a common language for biobanking. Eur J Hum Genet 2015;23:22-28.

2. Gasmelseed N, et al. Sub-Saharan centralized biorepository for genetic and genomic research. Sci Total Environ 2012; 423:210-213.
3. Gupta $S$, et al. Challenges and prospects for biomarker research: A current perspective from the developing world. Biochim Biophys Acta 2014;1844:899-908.

4. Astrin JJ, Betsou F. Trends in biobanking: A bibliometric overview. Biopreserv Biobank 2016;14:65-74.

5. Vaught J. Biobanking and biosecurity initiatives in Africa. Biopreserv Biobank 2016;14:355-356.

6. Mduluza T, et al. Maintaining respect and fairness in the usage of stored shared specimens. BMC Med Ethics 2013; 14(Suppl 1):S7.

7. Consortium HA, et al. Research capacity. Enabling the genomic revolution in Africa. Science 2014;344:13461348.

8. Gurdasani D, et al. The African Genome Variation Project shapes medical genetics in Africa. Nature 2015;517:327332.

9. Abayomi A, et al. Challenges of biobanking in South Africa to facilitate indigenous research in an environment burdened with human immunodeficiency virus, tuberculosis, and emerging noncommunicable diseases. Biopreserv Biobank 2013;11:347-354.

10. Mendy $\mathbf{M}$, et al. Infrastructure and facilities for human biobanking in low- and middle-income countries: A situation analysis. Pathobiology 2014;81:252-260.

11. Igbe MA, Adebamowo CA. Qualitative study of knowledge and attitudes to biobanking among lay persons in Nigeria. BMC Med Ethics 2012;13:27.

12. Staunton C, Moodley K. Challenges in biobank governance in Sub-Saharan Africa. BMC Med Ethics 2013;14:35.

13. Munung NS, et al. Obtaining informed consent for genomics research in Africa: Analysis of H3Africa consent documents. J Med Ethics 2016;42:132-137.

14. de Vries J, et al. The H3Africa policy framework: Negotiating fairness in genomics. Trends Genet 2015;31:117119.

15. Hewitt RE. Biobanking: The foundation of personalized medicine. Curr Opin Oncol 2011;23:112-119.

Address correspondence to: Elizabeth Sarah Mayne, MD

Department of Molecular Medicine and Haemotology University of the Witwatersrand National Health Laboratory Service 7 York Road Johannesburg 2050 South Africa

E-mail: elizabeth.mayne@nhls.ac.za 